\section{IGCP Projects 2007}

(OET — on extended term)

No. 467 Triassic time and trans-

Panthalassan correlations

Project leaders: M. J. Orchard (Canada),

L. Krystyn (Austria), J. Tong (China),

S. Lucas (United States), H. Campbell

(New Zealand), F. Hirsch (Japan),

K. Ishida (Japan), Y. Zacharov (Russia)

Duration: 2002-2006 (OET in 2007)

Website: http://paleo.cortland.edu/IGCP467/ index.html

No. 469 Late Variscan terrestrial biotas and palaeoenvironments

Project leaders: C.J. Cleal (United Kingdom), B.A. Thomas (United Kingdom),

S. Opluštil (Czech Republic), Y. Tenchov

(Bulgaria), E. Zodrow (Canada)

Duration: 2003-2007

Website: www.nmgw.ac.uk/www.php/301/

No. 473 GIS metallogeny of Central Asia

Project leaders: R. Seltmann (United Kingdom), A. Dolgopolova (Kazakhstan)

Duration: 2002-2006 (OET in 2007)

Websites: http://www.nhm.ac.uk/researchcuration/projects/cercams/index.html

http://www.nhm.ac.uk/mineralogy/cercams/ IGCP-473.html

\section{No. 474 Images of the Earth's Crust}

Project leaders: Bruce R. Goleby

(Australia), L.D. Brown (United States),

F. A. Cook (Canada), G.S. Fuis (United

States), R.W. Hobbs (United Kingdom),

D.M. Finlayson (Australia), Songlin Li

(China), Oncken (Germany)

Duration: 2003-2007

Website: http://www.earthscrust.org

No. 475 Deltas in the monsoon Asia-

Pacific region (DeltaMAP)

Project leaders: S. Goodbred, Jr. (United States), Y. Saito (Japan)

Duration: 2003-2007

Website: http://unit.aist.go.jp/igg/rg/cug-rg/ ADP/ADP_E/a_1stannual_en.html

No. 476 Monsoon evolution and tectonicclimate linkage in Asia

Project leaders: R. Tada (Japan), Hongbo

Zheng (China), Boo-Keun Khim (Korea),

P. Clift (United Kingdom), S. A. Gor-

barenko (Russia), B. N. Nath (India)

Duration: 2003-2007

Website: http://igcp.ees.hokudai.ac.jp/ 476

No. 478 Neoproterozoic-Early Palaeozoic events in Southwest Gondwana

Project leaders: C. Gaucher (Uruguay),

P. C. Boggiani (Brazil), A. Braun

(Germany), H. Frimmel (Germany), J.B.

Germs (South Africa), Poiré (Argentina)

Duration: 2003-2007

Official website:

www.vssagi.com/igcp478/igcp478.htm

Mirror website: www.igcp478.com

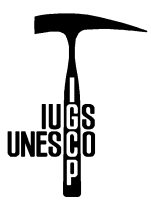

Related website: www.congresos-rohr.com/ vssagi/ingles/home.htm

No. 479 Sustainable use of platinum group elements

Project leaders: J. E. Mungall (Canada),

M. Iljina (Finland), C. Ferreira-Filho (Brazil) Duration: 2003-2007

Websites: http://www4.geology.utoronto.ca/ faculty/ mungall/Website/IGCP479Home .htm

http://gdr.nrcan.gc.ca/minres/data_e.php

No. 480 Tectonics of Central Asia

Project leaders: B. Natal'in (Turkey),

A. Yin (United States), A.M.C. Sengor

(Turkey), M. Kuzmin (Russia), Shuwen

Dong (China)

Duration: 2005-2009

Website: http://www.igcp.itu.edu.tr/index.html

No. 481 Dating Caspian sea level change

Project leaders: S.B. Kroonenberg (Nether-

lands), S. Leroy (United Kingdom)

Duration: 2003-2007

Websites: http://www.caspage.citg.tudelft.nl/ www.caspiansealevelchange.org

No. 482/489 Geodynamics of the East African rift system / geophysical characteristics and evolution of the southwestern branch of the East African rift system Project leaders: G. Mulugeta (Sweden)/

A. Atekwana (United States), M. P. Mod-

isi (Botswana), M. N. Sebagenzi (D. R.

Congo), J. J. Tiercelin (France)

Duration: 2003-2007

Website: http://w1.228.comhem.se/ u22800853

No. 485 The Boundaries of the West

African Craton

Project leaders: N. Ennih (Morocco), J-P. Liégeois (Belgium)

Duration: 2003-2007

Website: www.ucd.ac.ma/fs/igcp485/

No 486 Au-Ag-telluride-selenide deposits

Project leaders: N. J. Cook (Norway),

K. Kojonen (Finland)

Duration: 2003-2007

Website: www.ngu.no/igcp486

No. 487 Seismic microzoning of Latin

American cities

Project leaders: J. L. Alvarez Gómez (Cuba), A. Giesecke (Peru), G. F. Panza (Italy)

Duration: 2004-2008

Website: www.ictp.trieste.it/ sand/SMLAC/ SMLAC.html

No. 490 Environmental catastrophes Project leader: S. Leroy (United Kingdom) Duration: 2003-2007

Website:

http://www.mun.ca/canqua/igcp490/
No. 491 Middle Palaeozoic vertebrate biogeography, palaeogeography and climate

Project leaders: M. Zhu (P. R. China), G. Young (Australia)

Duration: 2003-2007

Website: http://paleoworld.net

No. 493 The rise and fall of the Vendian biota

Project leaders: M. Fedonkin (Russia) P. Vickers-Rich (Australia), J. Gehling (Australia)

Duration: 2003-2007

Website: http://www.earth.monash.edu.au/ PreCsite/index.html

No. 495 Quaternary land-ocean interactions

Project leaders: A. Long (United Kingdom), S. Islam (Bangladesh)

Duration: 2004-2008

Website: www.geography.dur.ac.uk/Projects/Default.aspx?alias=www.geography.dur.ac.uk/projects/igcp495

\section{No. 497 The Rheic Ocean}

Project leaders: U. Linnemann (Germany), R. D. Nance (United States), M. de Wit (South Africa), E. Bozkurt (Turkey),

P. Kraft (Czech Republic), F. Pereira (Portugal), R. A. Strachan (United Kingdom) Duration: 2004-2008

Website: http://www.snsd.de/igcp497/

No. 499 Devonian land-sea interaction: Evolution of Ecosystems and Climate in the Devonian

Project leaders: P. Königshof (Germany), J. Lazauskiene (Lithuania), E. Schindler (Germany), Volker Wilde (Germany) and N. Yalcin (Turkey)

Duration: 2004-2008

Websites: http://www.senckenberg.de/igcp-499 http://www.ipc2006.ac.cn

No. 500 Dryland change: past, present, puture

Project leader: D. Thomas (United Kingdom)

Duration: 2004-2008

No. 502 Global comparison of volcanichosted massive sulphide districts

Project leaders: R. Allen (Sweden),

F. Tornos (Spain), J. Peter (Canada),

N. Cagatay (Turkey)

Duration: 2004-2008

Website: www.ltu.se/tkg/avd/kgo/forsk/IGCP

No. 503 Ordovician palaeogeography and palaeoclimate Project leaders: T. Servais (France),

D.A. T. Harper (Denmark), J. Li (China),

A. Munnecke (Germany), W. Owen

(United Kingdom), P. M. Sheehan

(United States)

Duration: 2004-2008

Website: http://sarv.gi.ee/igcp503/

No. 506 Marine and non-marine Jurassic Project leaders: Jingeng Sha (China), Nicol Morton (France), W. A. P. Wimbledon (United Kingdom), Paul E. Olsen (United States), Alberto G. Riccardi (Argentina), 
Grzegorz (Gregory) Pien̆kowski (Poland) Yongdong Wang (China)

Duration: 2005-2006 (2009)

Website: http://www.nigpas.ac.cn/IGCP506

No. 507 Paleoclimates of the Cretaceous in Asia

Project leaders: Yong Il Lee (Korea), Xiaoqiao Wan (China), Takashi Sakai (Japan), and Krishnan Ayyasami (India)

Duration: 2006-2010

Website: http://igcp507.kopri.re.kr/

No. 508Y Volcano collapse and fault activity - "Young scientists project"

Project leaders: I. Alejandro Petrinovic

(Argantina), C. Corazzato (Italy),

T. Toulkeridis (Ecuador), A. Concha

Dimas (Mexico)

Duration: 2005-2007 (three years)

Website: http://www.geo.unimib.it/ IGCP508/ index.htm

No. 509 Palaeoproterozoic Supercontinents and Global Evolution

Project leaders: S. M. Reddy (Australia),

D. A. D. Evans (United States),

R. Mazumder (India)

Duration: 2005-2009

Website:

http://earth.geology.yale.edu/igcp509/

No. 510 A-type granites and related rock through time

Project leaders: Roberto Dall'Agnol

(Brazil), Carol D. Frost (United States),

O. Tapani Rämö (Finland), L. J. Robb

(South Africa)

Duration: 2005-2009

Website: http://www.igcp-510.org

No. 511 Submarine mass movements and their consequences

Project leaders: Jacques Locat (Canada), Juergen Mienert and Roger

Urgeles - (IOC link)

Duration: 2005-2009

Website: http://www.geohazards.no/IGCP511

No. 512 Neoproterozoic Ice Ages

Project leaders: Graham Shields (Australia), Emmanuelle Arnaud (Canada)

Duration: 2005-2009

Website: www.igcp512.com

No. 513 Karst aquifers and water resources

Project leaders: Chris Groves (United

States), Yuan Daoxian (China), Bar-

tolome Andreo-Navarro (Spain), Heather

Viles (United Kingdom)

Duration: 2005-2009

Websites: http://hoffman.wku.edu/igcp/ 513. html (General information)

http://aguas.igme.es/igme/AQUAiNMED /ing/defaultc.htm

http://www.hydrokarst.org/index.php?lang =en http://www.hydrokarst.org/mankarst.php?lang =en http://www.wku.edu/cehp

No. 514 Fluvial Palaeosystems: Evolution and Mineral Deposits

Project leaders: N. Patyk-Kara (Russia),

A. Duk-Rodkin (Canada), Baohong HOU
(Australia), Li Ziyang (China), Vladimir

Dolgopolov (Kazakhstan)

Duration: 2005-2009

Website: http://www.igem.ru/igcp514

No. 515 Coastal vulnerability related to sea level change

Project leaders: U. Simeoni (Italy), Maria Snoussi (Morocco), Zdravko Belberov

(Bulgaria), Francois Sabatier (France)

Duration: 2005-2009

No. 516 Geological anatomy of East and Southeast Asia

Project leaders: Ken-ichiro Hisada (Japan),

Punya Charusiri (Thailand), Byung-Joo

Lee (Rep. of Korea), Xiaochi Jin (China) Duration: 2005-2009

Website: http://staff.aist.go.jp/hara-hide/ igcp516

No. 519 Hydrogeology, hydrochemistry and management of coastal aquifers on the Atlantic coast of South America Project leaders: Emilia Bocanegra (Argentina), Emilio Custodio (Spain),

Marisol Manzano (Spain), Gerson

Cardoso (Brazil), Jenny Reynolds Vargas

(Costa Rica),

Duration: 2005-2009

No. 521 Black sea Mediterranean corridor during the last $30 \mathrm{ky}$ : Sea level change and human adaptation

Project leaders: Valentina Yanko-Hombach

(Canada), Yucel Yilmaz (Turkey), Pavel

Dolukhanov (United Kingdom)

Duration: 2005-2009

Websites : http://www.avalon-institute.org/ IGCP

http://www.bridge.bris.ac.uk/projects/

EMBSECBIO/index.html

http://www.sealevel.ns.ca/IGCP521/

No. 523 GROWNET - Global Ground

Water Network

Project leaders: Shrikant Daji LIMAYE

(India), Antony J Reedman (United Kingdom)

Duration: 2005-2009

Website: http://www.igcp-grownet.org/

No. 524 Arc-continent collision

Project leaders: Denis Brown (Spain), Chi-

Yue Huang (Taiwan)

Duration: 2007-2009.

No. 526 Risks resources and record of the past on the continental shelf

Project leaders: Francesco L. Chiocci

(Italy), Lindsay Collins (Australia),

Michel Michaelovitch de Mahiques

(Brazil), Renée Hetherington (Canada)

Duration: 2007(-2011)

No. 529 Availability of groundwater resources in selected urban areas in Southern African Development Community (SADC) region

Project leader: Imasiku A. Nyambe (Zambia)

Duration: 2007-2011
No. 534 Reconstruction of the past coastal environments and its management Project leaders: Marta Pappalardo (Italy),

Florina Grecu (Romania)

Duration: 2007(-2011)

No. 540 Gold-bearing hydrothermal fluids of orogenic deposits

Project leaders: P.S. Garofalo (Italy),

J. R. Ridley (United States), Vsevolod

Prokofíev (Russia)

Duration: 2007-2011

No. 543 Low-temperature thermochronology: applications and interlaboratory calibration

Project leaders: Massimiliano Zattin (Italy),

J. I. Garver (United States), Vitaliy

A. Privalov (Ukraine), Alexei V.

Soloviev (Russia), Cornelia Spiegel (Germany), Maarten de Wit (South Africa),

Dewen Zheng (China)

Duration: 2007-2010

No. 545 Clays and clay minerals in

Africa

Project leader: Georges-Ivo E. Ekosse

(South Africa)

Duration: 2007-2011

No. 546 Subduction zones of the

Caribbean

Project leaders: Antonio Garcia-Casco

(Spain), Uwe Martens (United States)

Duration: 2007-2011

No. 555 Rapid environmental/climate change in the Cretaceous greenhouse world

Project leaders: Chengshan Wang (China), Robert Scott (United States), Hugh Jenkyns (United Kingdom), Michael Wagreich (Austria), William Hay (United States); Zakharov Y.D. (Russia)

Duration: 2007-2010

No. 557 Diamonds, xenoliths and kimberlites

Project leaders: Holger Sommer

(Botswana), Klaus Regenauer-Lieb (Australia), Christoph Hauzenberger (Austria), Jonathan Kashabano (Tanzania), Gétan

Moloto-A-Kenguemba (Central African Republic)

Duration: 2007-2011

Funded projects

O.E.T.

Total

updated 23/03/2007

\section{IGCP Secretariat}

UNESCO, Division of Ecological and Earth Sciences

1, Rue Miollis

75732 Paris Cedex 15

FRANCE

Tel: +33145684118

Fax: +33145685822

www.unesco.org/sciencelearth

E-mail:igcp@unesco.org 